\title{
Biología de \\ Lycoriella ingenua y Bradysia impatiens (Diptera: Sciaridae)
}

\section{Biology of Lycoriella ingenua and Bradysia impatiens (Diptera: Sciaridae)}

\author{
Victor H. Marín-Cruz', David Cibrián-Tovarl, ", José T. Méndez-Montiel', Omar A. Pérez-Veral, \\ José A. Cadena-Meneses ${ }^{2}$, Herón Huerta ${ }^{4}$, Gabriel Rodríguez-Yam y Juan A. Cruz-Rodríguez ${ }^{3}$
}

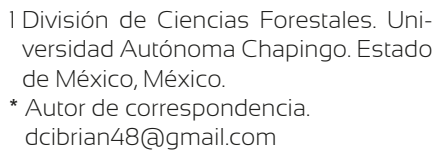

1 División de Ciencias Forestales. Universidad Autónoma Chapingo. Estado de México, México.

* Autor de correspondencia. dcibrian48@gmail.com

\author{
2 Departamento de Zootecnia. Univer- \\ sidad Autónoma Chapingo. Estado \\ de México, México. \\ cadena(a)correo.chapingo.mx
}

\author{
3 Departamento de Agroecología. Uni- \\ versidad Autónoma Chapingo. Esta- \\ do de México, México.
}

4 Laboratorio de Entomología. Instituto de Diagnóstico y Referencia Epidemiológico. Secretaria de Salud. Distrito Federal, México. cerato_2000@yahoo.com

\section{RESUMEN}

Se estudiaron dos especies de mosquito fungoso negro, Lycoriella (Lycoriella) ingenua (Dufour, 1839) y Bradysia impatiens (Johannsen, 1912) (Diptera: Sciaridae), que causan daño a plántulas de Pinus montezumae (Lamb, 1832) en viveros forestales. Estos dípteros se identificaron morfológicamente y por métodos moleculares y se citan por primera vez como plaga en viveros forestales de México. La proporción sexual para L. ingenua fue de un macho por cada 1.25 hembras; para caracterizar esta especie se midieron huevos, pupas, larvas y cápsulas cefálicas. Con los datos morfométricos de longitud de cuerpo y ancho de la cápsula cefálica de larvas y mediante el método de conglomerados jerárquicos se determinaron los estadios larvarios de la especie. Para B. impatiens por cada macho existen 2.4 hembras. En la distinción de los estadios larvarios, el mejor parámetro fue el ancho de cápsula cefálica. Ambas especies presentaron cuatro estadios larvarios. Las dos especies se criaron en laboratorio a $21^{\circ} \mathrm{C} \pm 2{ }^{\circ} \mathrm{C}$. El ciclo completo de L. ingenua requirió de 30.5 días \pm 3.5 días. Para B. impatiens el tiempo para concluir ciclos biológicos fue de 27.5 días \pm 2.5 días. Se determinó que las larvas de ambas especies consumen la raíz de Pinus montezumae. Se identificaron los hongos asociados a larvas y adultos de ambas especies, Fusarium circinatum fue el más importante y se registra por primera vez en viveros de P. montezumae; esta especie de hongo se identificó mediante morfología y por métodos moleculares.

Palabras clave: ciclo biológico, estadios larvarios, hongos asociados, mosquito fungoso negro, proporción de sexos.

\section{ABSTRACT}

Lycoriella (Lycoriella) ingenua (Dufour, 1839) and Bradysia impatiens (Johannsen, 1912) (Diptera: Sciaridae) are two black fungus gnats species studied that harm Pinus montezumae (Lamb, 1832) seedlings in forestry nurseries. These dipterous were morphological and molecularly identified and are mentioned for the first time as a pest in Mexico's forestry nurseries. The proportion of sexes to Lycoriella ingenua was of one male per 1.25 females; to characterize this species eggs, pupas, larvae and cephalic capsules were measured. The morphometric data of body length and cephalic cage width of larvae through the hierarchical conglomerated method determined the larval instars of the species. For B. impatiens there are 2.4 females per male. In the definition of the larval instars, the best parameter was that of the cephalic capsule. Both species presented four larval instars. Both species were bred under lab conditions at $21^{\circ} \mathrm{C} \pm 2{ }^{\circ} \mathrm{C}$. The whole cycle of $L$. ingenua required $30.5 \pm 3.5$ days. For B. impatiens the time to conclude biological cycles was of $27.5 \pm 2.5$ days. It was determined that both species of larvae consume Pinus montezumae root. The fungi associated to larvae and fungi of both species were identified. Fusarium circinatum was the most important and it is registered for the first time in nurseries of Pinus montezumae; this species was morphological and molecularly identified.

KEY wORDs: biological cycle, larval instars, associated fungi, black fungus gnats, sex proportion. 


\section{INTRODUCCIÓN}

Los adultos de la familia Sciaridae son pequeños dípteros que miden 1 mm - 6 mm (Mohrig y Menzel, 2009); conocidos comúnmente como moquitos fungosos negros, representados por varias especies de diferentes géneros, las cuales son consideradas como plagas en viveros e invernaderos. Dentro de estos géneros se incluye Lycoriella Frey, 1972 y Bradysia Winnertz, 1867. Las larvas de estos insectos se alimentan de hongos, algas y materia orgánica en descomposición, eventualmente bajo ciertas circunstancias son capaces de perforar raíces sanas de diferentes especies y semillas carnosas, y en infestaciones severas ocasiona la muerte de la planta (Aguilera y Ortega, 1996; Cibrián et al., 2008; Landis et al., 1989; Mansilla et al., 2001; Springer, 1995a, 1995b). Varias especies de la familia Sciaridae son documentadas como plagas de importancia económica en invernaderos de ornamentales, hortícolas y forestales, y en la producción del hongo Agaricus bisporus (Lange) Imbach, 1946 (Erler et al., 2011; Landis et al., 1989; Loudon, 1978; O'Connor y Keil, 2005; Radin et al., 2009; Shin et al., 2012; Smith et al., 2006; Steffan, 1981; White et al., 2000). Las plantas atacadas por las larvas del mosquito fungoso negro manifiestan síntomas como pérdida de vigor, marchitez, desprendimiento de hojas, escaso crecimiento y amarillamiento de follaje (Cibrián et al., 2008, Landis et al., 1989; Pundt, 1999). Además, las larvas y adultos de mosquito fungoso negro pueden facilitar la infección de plántulas al llevar sobre su cuerpo esporas de hongos patógenos como Pythium (Braun et al., 2009; Gardiner et al., 1990), Fusarium (Gillespie y Menzies, 1993; Hurley et al., 2007; Keates et al., 1989), Botrytis (James et al., 1995; Keates et al., 1989), Verticillium (Kalb y Millar, 1986) y Phoma (Keates et al., 1989).

En los últimos años se ha encontrado al mosquito fungoso negro alimentándose de las raíces de plántulas de árboles como eucaliptos y coníferas (Cibrián et al., 2008; James et al., 1995; Hurley et al., 2007; Keates et al., 1988; Landis et al., 1989; Mansilla et al., 2001; Menzel et al., 2003, 2006). Cibrián et al. (2008) encontraron infestaciones severas de mosquito fungoso en viveros forestales en el centro de México, causando la perdida de hasta 30\% de plántulas de coníferas.

En el ciclo de producción de planta forestal de 20102011, en el vivero forestal de Temamatla, Estado de México se tuvieron infestaciones severas por ciáridos, con pérdidas de más de $30 \%$ de la cosecha de planta (1.5 millones de un total de 6 millones) (Cibrián, datos no publicados). La información básica para el manejo de la plaga era reducida e incompleta, no se conocían las especies de mosquitos fungosos involucrados, ni sus ciclos y hábitos.

\section{OBJETIVOS}

- Identificar las especies de mosquito fungoso negro en el vivero de Temamatla, Estado de México.

- Determinar en laboratorio el ciclo biológico del mosquito fungoso negro.

- Conocer la biología de las especies de ciáridos relacionadas con las plántulas de Pinus montezumae y los hongos asociados a especies de estos insectos en el vivero forestal de Temamatla, Estado de México.

\section{MATERIALES Y MÉTODOS}

\section{Recolecta del material entomológico}

Se realizaron recolectas de adultos y larvas de mosquito fungoso negro durante el mes de octubre de 2012, en plántulas de dos meses de edad de Pinus montezumae, del vivero forestal de Temamatla, Estado de México. Los adultos de mosquito fungoso negro se capturaron con un aspirador manual. Las larvas se recolectaron con un pincel directamente de la raíz y del sustrato donde crece el pino. Las recolectas se realizaron en un solo día y fueron sistemáticas a cada tres metros entre recolecta en una platabanda de 21 metros de longitud.

\section{Identificación de especies del mosquito fungoso negro}

Los adultos recolectados fueron separados por morfoespecies de ciáridos, de ellos 50 ejemplares se sacrificaron y conservaron en frascos con etanol al 70\%. Con un microscopio estereoscopio Leica se separaron por sexo. Posterior- 
mente, a 20 machos se les realizaron cortes de los genitales, cabeza, palpos, tibias y alas, estas estructuras se prepararon en laminillas con azul de algodón para aclarar los tejidos (Poinar y Thomas, 1984). Para su estudio se usó un microscopio óptico Leica M80 y una cámara modelo DFC295 (Software Leica Application Suite V3.0 ${ }^{\circledR}$ ) de la misma marca; se tomaron fotografías de las estructuras importantes para la identificación de especies (todas las mediciones en este estudio se realizaron con un microscopio y software). La identificación se realizó con las claves taxonómicas de la familia Sciaridae de Mohrig y Menzel (2009), Steffan (1981), Komarov (2009), Menzel et al. (2003), Mohrig et al. (2012) y Shin et al. (2012). La identificación morfológica de las especies se corroboró con un estudio molecular con la amplificación de una región del gen COI del ADNmt por medio de la PCR usando los oligonucleótidos CI-J-1751 and CI-N-2191 (Hurley et al., 2010). El producto de la PCR se secuenció en Macrogen (Corea), se analizó y se depositó en el GenBank.

\section{Ciclo biológico y comportamiento de ciáridos}

\section{Ciclo biológico}

Los adultos de las dos especies previamente identificadas, se colocaron por separado en cámaras de cría. Las cámaras de cría consistieron en recipientes de plástico transparente de medio litro de capacidad. Las tapas se perforaron en el centro (círculo de $3 \mathrm{~cm}$ de diámetro) para introducir los adultos de mosquito fungoso negro y se sellaron con un tapón de algodón para evitar que se salieran. Se colocaron diez repeticiones por especie, cada una consistió en 10 hembras y dos machos. Los envases de plástico se prepararon con recortes de papel bond blanco que cubrían el fondo y tres rodajas de papa $(3 \mathrm{~cm}$ de diámetro y $1.5 \mathrm{~cm}$ de alto) previamente flameadas con alcohol al $70 \%$. A cada rodaja se le realizaron cuatro ranuras de $0.5 \mathrm{~cm}$ de profundidad con una aguja de disección, con la finalidad de que las hembras ovipositaran sus huevos en ellas. Para mantener la humedad en los recipientes, cada cuatro días se agregaron $3 \mathrm{ml}$ de agua destilada. Las cámaras de cría se mantuvieron a una temperatura $21^{\circ} \mathrm{C} \pm 2{ }^{\circ} \mathrm{C}$.
Tras la oviposición de los huevos, estos se sacaron y se colocaron en grupos de 30-50 en cajas Petri (9 cm de diámetro), preparadas con el fondo de papel bond y una rodaja de papa. Se registró el desarrollo en días de los estados biológicos de ambas especies, hasta que inició la emergencia de nuevos adultos. Los adultos recién emergidos se separaron en parejas, macho y hembra; cada pareja se colocó en una nueva caja Petri, de esta manera se aseguró el desarrollo de cada especie; se llevaron a cabo 15 repeticiones por cada especie, se tomaron datos todos los días del desarrollo de cada estadio hasta terminar el ciclo. La duración del ciclo biológico se determinó a partir de la aparición de nuevos adultos. Los adultos muertos se prepararon sobre un portaobjetos con azul de algodón y se observaron al microscopio compuesto para determinar la especie.

\section{Huevos}

Para determinar el número de huevos ovipositados por hembra, en un envase de plástico de medio litro de capacidad (preparado de la misma forma que los anteriores), se colocaron un macho y una hembra de la misma especie recién emergidos sin aparearse (15 repeticiones). Tras la oviposición y una vez que la hembra murió se observaron las rodajas de papa en un microscopio óptico estereoscópico. Se contó el número de huevos ovipositados por hembra y se describió la forma en la que se ovipositaron y agruparon. Se midió la longitud y anchura de 66 huevos.

\section{Estadios larvales}

La separación de estadios larvales se realizó por medio de la disposición de los orificios ventiladores, como lo indica Steffan (1981). Las larvas de la familia Sciaridae tienen cuatro estadios. Para confirmar la definición de estadios se hicieron estudios morfométricos por cada especie; en 182 larvas de L. ingenua se midió el largo y el ancho del cuerpo y longitud de la cápsula cefálica; en 94 larvas de $B$. impatiens se midió la longitud y anchura de la cápsula cefálica (se hicieron pruebas preliminares mediante el análisis de conglomerados jerárquicos y con la medidas tomadas a cada especie se obtuvo la mejor clasificación). Los datos de morfometría se analizaron mediante el Aná- 
lisis de Conglomerados Jerárquicos (V 6.1, 2001). Este procedimiento Análisis Multivariante está diseñado para encontrar agrupaciones naturales (conglomerados o " $c l u s$ ters") dentro de un conjunto de datos, se basa en el supuesto de que los datos son generados por una distribución-mezcla. Este procedimiento está implementado en la función "mclust" del S-Plus (V 6.1, 2001). Para cada número posible de grupos este procedimiento obtiene la mejor clasificación y proporciona los valores AWE( $k$ ) (por sus siglas en inglés), obtenidos como una función del factor de Bayes del modelo sin agrupamiento $v s$ la clasificación con $k$ grupos. Para seleccionar el número de grupos con base en este criterio se puede seleccionar el valor $k^{*}$ que tiene valor AWE máximo. Sin embargo, si AWE $\left(k^{*}-1\right)$ no difiere mucho de $\operatorname{AWE}\left(k^{*}\right)$, puede ser preferible $k^{*}-1$ por el principio de parsimonia.

\section{Pupas}

Se medió la longitud y la anchura de 24 pupas, se determinó el tiempo que tardan en emerger los adultos, además se observó y se documentó el comportamiento del cambio de coloración de las pupas.

\section{Proporción de sexos}

La proporción de sexos se determinó por medio de los genitales de los adultos de ambas especies de mosquito fungoso negro, 750 individuos de L. ingenua y 583 de $B$. impatiens. Para obtener los individuos se colocaron en cámaras de cría 10 hembras y dos machos por especie; se realizaron cinco repeticiones por cada especie. Las cámaras de cría consistieron en envases de plástico de medio litro de capacidad y fueron preparadas de igual forma que las utilizadas en el apartado de ciclo biológico. Una vez que se obtuvieron nuevos adultos, estos se recolectaron, se sacrificaron y se preservaron en alcohol al $70 \%$, para su posterior separación de machos y hembras.

\section{Comportamiento de los ciáridos}

Se recolectaron plántulas de pino que presentaron síntomas de ataque de mosquito fungoso negro. El cepellón de las plántulas fue removido con agua corriente. Mediante observaciones al microscopio estereoscópico se revisaron las raíces que dieron positivo a la presencia de daños de larvas y se identificó el sitio de entrada de la larva a la raíz. Después, se realizó un corte longitudinal a cada raíz del cuello de la raíz hacia bajo. Los daños causados por las larvas de mosquito fungoso negro en cada raíz fueron descritos. En cajas Petri (9 cm diámetro) se colocaron en un extremo 10 larvas de tercer estadio de mosquito fungoso negro y en el extremo opuesto de la caja Petri se colocó una plántula de pino de $4 \mathrm{~cm}$. Las larvas y la plántula se colocaron con una separación de $7.5 \mathrm{~cm}$. Se contabilizó el tiempo que tardaron las larvas en detectar la raíz y llegar a ella. Este proceso se realizó con 15 repeticiones.

Para L. ingenua se observó el comportamiento de larvas y adultos en las cámaras de cría. Se registró día con día el hábito de alimentación, postura de huevos, formación de puparios, apareamiento, duración de estadios y ciclo completo, para conocer en forma precisa sus hábitos en laboratorio.

\section{Hongos asociados al mosquito fungoso negro}

Para determinar los hongos asociados al cuerpo del mosquito fungoso negro, se recolectaron al azar de 20 contenedores con P. montezumae, los adultos se recolectaron con un aspirador manual y las larvas con un pincel. En un lote de 10 cajas Petri (9 $\mathrm{cm}$ de diámetro) con medio de cultivo de agar Dextrosa-Saboraud sin antibiótico se colocaron 10 larvas vivas por cada caja; en otro lote de cajas Petri preparadas igual que las anteriores, se colocaron 10 adultos vivos por cada caja. Las cajas Petri se mantuvieron a una temperatura de $23{ }^{\circ} \mathrm{C} \pm 2{ }^{\circ} \mathrm{C}$. Una vez que los adultos padres murieron se recolectaron y se corroboró a que especie pertenecían. De igual forma, cuando los nuevos adultos murieron, se recolectaron y se identificó la especie. Las cajas Petri se revisaron diariamente para ver el crecimiento de los hongos, al octavo día, las colonias de hongos que crecieron se reaislaron para tener cultivos puros. Los géneros de hongos se identificaron con la clave de Carrillo (2003), se utilizó un microscopio óptico Leica. La caracterización molecular de Fusarium sp., se realizó una amplificación de un fragmento de las regiones inter- 
nas ITS-PCR con los oligonucleótidos específicos CIRC1A y CIRC4A (Hurley et al., 2007). El producto de PCR se secuenció en Macrogen (Corea), se analizó y se depositó en el GenBank.

\section{RESULTADOS Y DISCUSIÓN}

\section{Identificación de especies}

Se encontraron las especies Bradysia impatiens y Lycoriella ingenua como agentes causales de mortalidad de plántulas de Pinus montezumae. Para B. impatiens se utilizaron las claves taxonómicas de Steffan (1981) y Mohrig y Menzel (2009); así como la redescripción de Menzel et al. (2003), Shin et al. (2012) y Mohrig et al. (2012). Con respecto a Lycoriella se utilizaron las claves de Komarov (2007), Shin et al. (2012) y Mohrig et al. (2012). De acuerdo con Mohrig et al. (2012), en su reciente revisión de la familia Sciaridae de Norte América se acepta que $B$. difformis es sinónimo de B. impatiens (Johannsen).

\section{Diagnosis de Bradysia impatiens (Johannsen, 1912)}

Adulto: Macho. Color pardo oscuro a negro, longitud $2.25 \mathrm{~mm}$. Cabeza: Puente ocular con 2 a 3 facetas de ancho. Antena corta comprimida y uniformemente oscura, longitud $1.27 \mathrm{~mm}$; cuarto flagelómero (Fig. 1A) 1.6 veces más largo que ancho, superficie ligeramente rugosa. Palpo (Fig. 1B) moderadamente largo, amarillo a pardo claro, con tres segmentos, segmento basal con la fosa sensorial profunda, sensilas largas, ligeramente curvadas con punta roma; siete sedas largas dispuestas sobre el segmento. Tórax pardo oscuro a negro, con áreas laterales pardo claras a amarillas. Coxas y fémures pardos claros a blanquecinos amarillo; tibias y tarsos oscurecidos por revestimiento de las sedas oscuras y gruesas. Postpronoto sin sedas. Mesonoto con sedas largas y laterales y sedas cortas dispersas sobre la superficie (setulae). Katepisterno triangular. Escutelo con tres sedas largas. Lado interno de la tibia anterior con una hilera de 10 sedas (Fig. 1C); tibia media y posterior con dos sedas delgadas en forma de espolones, subiguales. Uñas tarsales sin dientes.
Ala (Fig. 1D) con longitud total $1.95 \mathrm{~mm}$, anchura 0.80 $\mathrm{mm}$, infuscada grisácea-parda; venas posteriores sin macrotriquias; base de la $\mathrm{M}$ más larga que la bifurcación de M. Genitales compactados (Fig. 1E); sin lóbulo basal o grupo de sedas en la vista ventral. Gonocoxito corto, cubierto con sedas oscuras, así como sedas gruesas y largas principalmente en la base. Tergito 9 corto, trapezoidal, ligeramente emarginado apicalmente con varias sedas largas. Gonostilo (Fig. 1F) 2.5 veces más largo que ancho, ocho espinas subiguales curvadas ventromedialmente y una espina apical gruesa. Tegmen ligeramente más ancho que largo, redondeado apicalmente; edeago con base esclerotizada, longitud $0.1 \mathrm{~mm}$.

Hembra. Similar al macho. Longitud $2.25 \mathrm{~mm}$. Antena longitud $1.30 \mathrm{~mm}$. Cuarto flagelómero 2.2 veces más largo que ancho. Palpo con el segmento basal con la fosa sensorial profunda y oscura, 7 sedas largas dispuestas sobre el segmento. Longitud del ala $2.8 \mathrm{~mm}$.

Esta es la primera vez que se documenta la especie $B$. impatiens en viveros forestales de México. Se extrajo del interior de raíces de plántulas de P. montezumae. Un total de 87 adultos de $B$. impatiens fueron colectados del vivero de Temamatla, México. De los cuales tres machos fueron procesados molecularmente; las secuencias genéticas se depositaron en el GenBank con el número de acceso KP120771. Según Mohring et al. (2012), B. impatiens ha sido diseminada por el hombre con el movimiento plantas. Esta especie es común en macetas, materia orgánica, jardines e invernaderos (Menzel et al., 2006; Mohring et al., 2012; Shin et al., 2012). Su distribución es mundial, desde Sudáfrica, Brasil, Hawai, Republica Checa, Finlandia, Alemania, Gran Bretaña, Italia, Holanda, Rusia, España, Suiza, Letonia Azerbaiyán, Japón, Corea, Canadá, E. U. (Menzel et al., 2003; Mohring et al., 2012; Shin et al., 2012). En México $B$. impatiens es conocida como plaga común en los invernaderos de nochebuena (Euphorbia pulcherrima Willd. ex Klotzch) (García, 2008). En Gran Bretaña se ha encontrado atacando hongos comestibles en invernaderos (White et al., 2000). Mansilla et al. (2001) la hallaron causando daño en viveros de Eucalyptus en Italia. Hurley et al. (2007) y (2010) identificaron a B. difformis en viveros de 

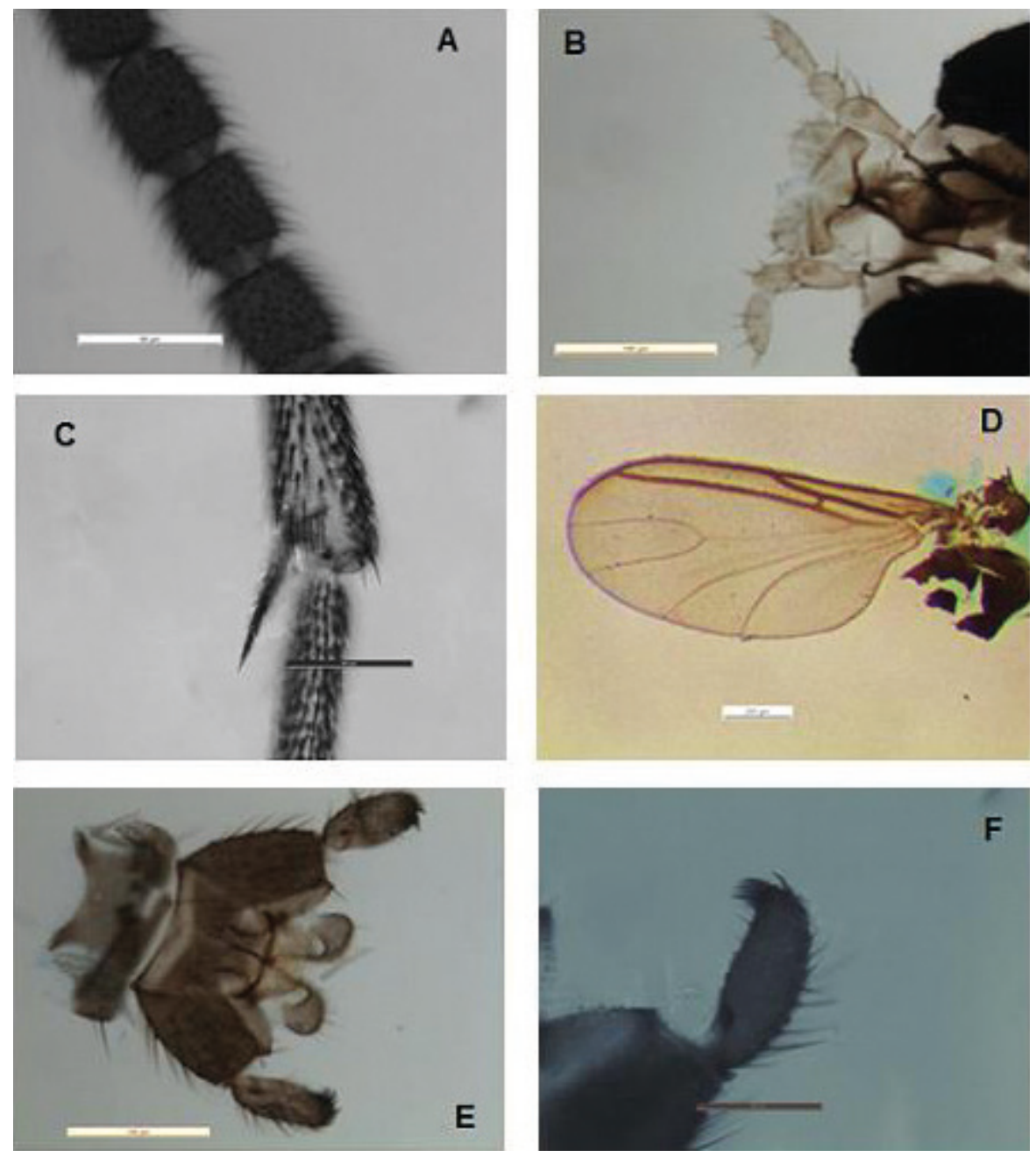

Figura 1. Macho de B. impatiens (A-F). (A) Cuarto flagelómero.

(B) Palpo. (C) Tibia anterior, peine apical. (D) Ala. (E) Genitales, vista ventral. (F) Gonostilo, vista lateral.

coníferas en Sudáfrica. Por su amplia distribución esta especie es diseminada por las actividades de cultivo del humano, no solo por medio de las plantas, sino también a través de los sustratos, específicamente en la turba.

\section{Diagnosis de Lycoriella (Lycoriella) ingenua (Dufour, 1839)}

Macho (Fig. 2A). Cabeza: puente ocular con 4 facetas de ancho. Antena uniformemente oscura, longitud $1.48 \mathrm{~mm}$; cuarto flagelómero 2.1 veces más largo que ancho (Fig. 2B), cuello corto pero distinguible. Palpo pardo claro, con tres segmentos, segmento basal con la fosa sensorial profunda, 8 sedas dorsales largas y dispuestas sobre el seg- mento (Fig. 2C). Tórax oscuro. Coxas y fémures pardo-claros; tibias y tarso oscurecidos por el revestimiento de las sedas oscuras y gruesas. Mesonoto con sedas largas y sedas cortas dispersas sobre la superficie (setulae). Katepisternon triangular. Escutelo con sedas largas. Lado interno de la tibia anterior compuesta de 14 sedas (Fig. 2D); tibia media y posterior con dos sedas delgadas en forma de espolones, subiguales. Uñas tarsales simples. Ala longitud total $2.35 \mathrm{~mm}$, anchura $0.84 \mathrm{~mm}$ (Fig. 2E); venas posteriores sin macrotriquias; base de la $\mathrm{M}$ más larga que la bifurcación de M. Genitales (Fig. 2F). Gonocoxito con lóbulo basal compuesto de 14 sedas. Tergito 9 triangular. Gonostilo (Fig. 2H) 3.3 veces más largo que ancho, ápice 

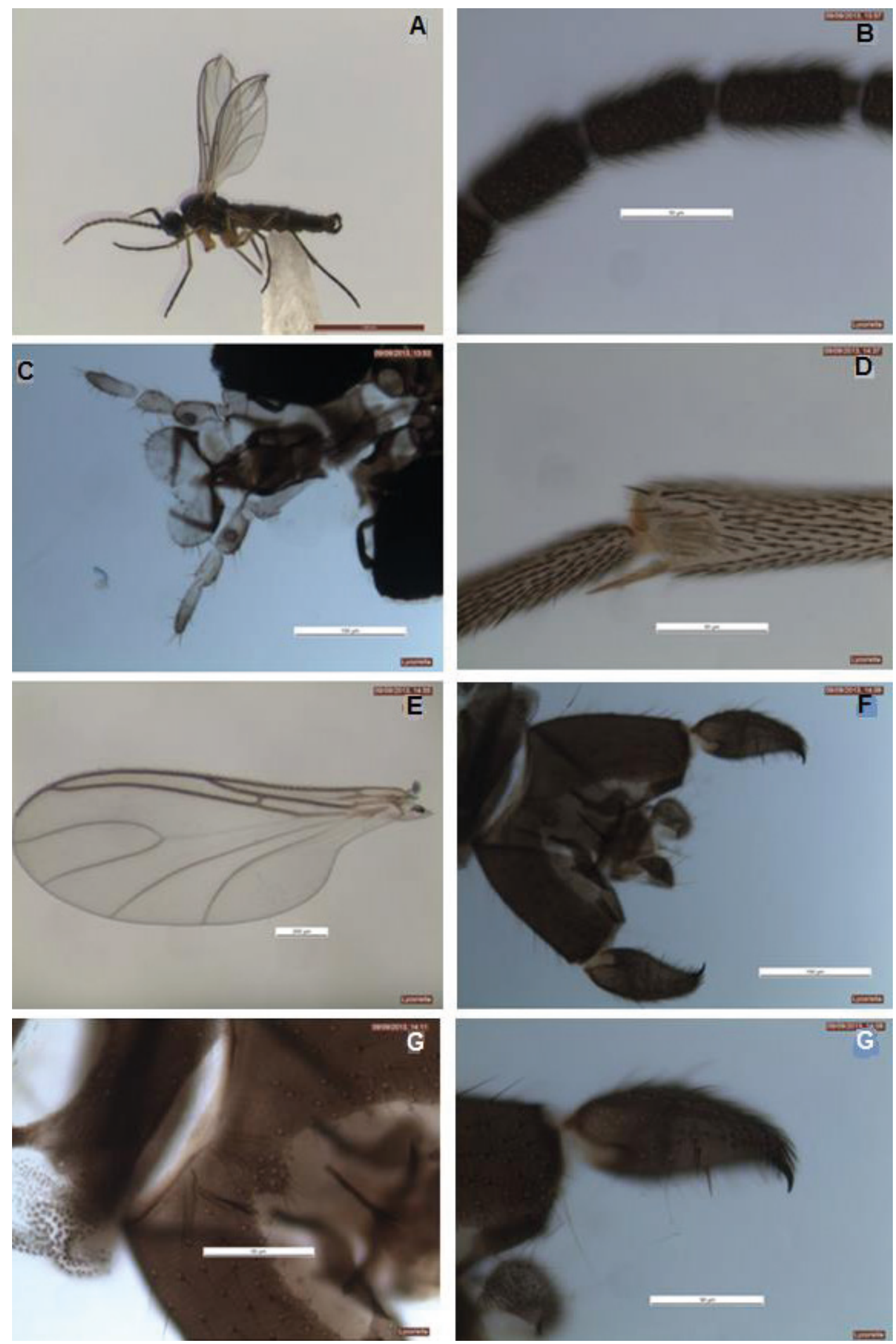

Figura 2. Macho de L. ingenua (A-H). (A) Macho, hábito. (B) Cuarto flagelómero, vista lateral. (C) Palpo. (D) Tibia anterior, peine apical. (E) Ala. (F) Genitales, vista ventral. (G) Lóbulo basal del gonocoxito, vista ventral. (H) Gonostilo, vista lateral. 
con sedas gruesas, curvadas ventromedialmente y varias sedas mediales a lo largo del margen interno, una seda larga, en forma de látigo cerca de la base; espina apical robusta y curvada, conspicua (Fig. 2G). Tegmen más ancho que largo, ápice ligeramente emarginado; edeago con base esclerotizada, longitud $0.06 \mathrm{~mm}$.

Hembra. Similar al macho. Longitud $3.0 \mathrm{~mm}$. Antena longitud $1.25 \mathrm{~mm}$. Cuarto flagelómero 2.28 veces más largo que ancho. Palpo con el segmento basal con la fosa sensorial profunda y oscura, y 8 sedas largas dispuestas sobre el segmento. Longitud del ala $2.38 \mathrm{~mm}$.

En este estudio $L$. ingenua se documenta por primera vez en viveros forestales de México, causando daño a plántulas de P. montezumae. Sin embargo, Pérez et al. (2013), encontraron esta especie en México como plaga en la producción de Agaricus bisporus (Lange) Imbach en Perote, Veracruz. L. ingenua es una plaga común en el cultivo de hongos y en invernaderos, la especie puede encontrarse también en suelos forestales y en áreas agrícolas (Mohring, et al., 2012). En el mundo L. ingenua es considerada una plaga importante en la producción del hongo A. bisporus (Erler et al., 2011; O'Connor y Keil, 2005; Shamshad et al., 2009; White et al., 2000). Esta especie se distribuyen en Asia (Japón y Korea), Europa (Polonia, Gran Bretaña), Norte América, México, Rusia, Turquía, Australia. Probablemente $L$. ingenua tenga una distribución cosmopolita (Erler et al., 2011; Komarov, 2007; Lewandowski et al., 2004; Mohring, et al., 2012; Pérez et al., 2013; Shamshad et al., 2008; Shin, et al., 2012). Se recolectaron 48 adultos de L. ingenua de las cámaras de cría, De los cuales cuatro machos fueron procesados molecularmente; las secuencias genéticas fueron depositadas en el GenBank con número de acceso KP100538.

\section{Ciclos biológicos y comportamiento de L. ingenua y B. impatiens}

\section{Ciclo biológico}

El ciclo de vida completo de B. impatiens tuvo una duración de 27.5 días (intervalo de 25 días a 30 días, $\mathrm{n}=11$ ciclos) en condiciones de laboratorio a una temperatura de $21^{\circ} \mathrm{C} \pm 2^{\circ} \mathrm{C}$. La duración en cada estadio de desarrollo fue en huevo 4.5 días (rango 4-5 días), larva de primer estadio 2 días, larvas de segundo estadio 2.5 días (rango 2-3 días), larva de tercer estadio 4 días (rango 3.5-4.5 días), larva de cuarto estadio 4 días (rango 3-5 días); la duración de los cuatro estadios larvarios fue de 12.5 días (rango 11-13 días), prepupa 1 día, pupa 4 días y adulto 5.5 días (rango 4.5-6.5 días). Wilkinson y Daugherty (1970) encontraron una duración igual (27.5 días) del ciclo biológico para $B$. impatiens a una temperatura de $24^{\circ} \mathrm{C}$ y la duración de los cuatro instares larvarios es de 13.1 días. Además, los resultados obtenidos en este trabajo son similares a los obtenidos por Mansilla et al. (2001), quienes encontraron un ciclo biológico de $B$. impatiens (temperatura de $23{ }^{\circ} \mathrm{C} \pm 1{ }^{\circ} \mathrm{C}$ ) de 21 a 28 días (24 días en promedio) y la duración de estadios larvarios es de 9-13 días. La diferencia entre la duración de los ciclos de vida obtenida en este trabajo y por Wilkinson y Daugherty (1970), con respecto con Mansilla et al. (2001), se puede deber a el uso de diferentes temperaturas en la cría en laboratorio, en este estudio la temperatura fue menor 2 ${ }^{\circ} \mathrm{C}$ y el ciclo fue mayor en tres días.

Por otro lado el ciclo biológico de L. ingenua, bajo las mismas condiciones que $B$. impatiens, tuvo una duración de 30.5 días (rango 27-34 días, $\mathrm{n}=12$ ciclos). En la tabla 1 se presentan diferentes duraciones del ciclo de L. ingenua. La duración de los diferentes estadios larvarios son: primer estadio 2.5 días (rango 2-3 días); segundo y tercer estadio presenta una duración similar 4 días (rango 3.54.5 días), cuarto estadio 6 días (rango 5-7 días).

Frouz y Nováková (2001), concluyeron que la duración del ciclo biológico de L ingenua depende directamente de la temperatura, lo cual puede variar de 18 a 40 días con una temperatura respectivamente de $25{ }^{\circ} \mathrm{C}$ y $15{ }^{\circ} \mathrm{C}$. La duración del ciclo biológico de L. ingenua es dependiente de la temperatura y de las dietas con que se alimentan.

\section{Huevo}

El huevo de L. ingenua. Liso y blando. Longitud en promedio $0.213 \mathrm{~mm}$ y $0.134 \mathrm{~mm}$ de ancho (rango 0.091$0.143 \mathrm{~mm}, \mathrm{DE}=0.0157, \mathrm{n}=66)$. Recién ovipositado de color blanco lechoso y conforme se desarrolla se torna de 
Madera y Bosques $\quad$ vol. 21, núm. 1: 113-128 Primavera 2015

Tabla 1. Ciclos biológicos de L. ingenua de autores y temperaturas diferentes.

\begin{tabular}{ccccc}
\hline $\begin{array}{c}\text { Duración promedio de } \\
\text { estadio en días }\end{array}$ & $\begin{array}{c}\text { Datos originales } \\
21{ }^{\circ} \mathrm{C} \pm 2{ }^{\circ} \mathrm{C}\end{array}$ & $\begin{array}{c}{ }^{*} \text { Steffan (1974) } \\
20{ }^{\circ} \mathrm{C} \pm 2{ }^{\circ} \mathrm{C}\end{array}$ & $\begin{array}{c}\text { Lewandowski et al. (2004) } \\
24{ }^{\circ} \mathrm{C}\end{array}$ & $\begin{array}{c}\text { Frouz y Nováková (2001) } \\
2{ }^{\circ} \mathrm{C}\end{array}$ \\
\hline Huevo & 4 & 3.3 & 3 & 3.5 \\
Larva estadios I-IV & 16.5 & 13.6 & 13 & 18.5 \\
Pupa & 5 & 3.1 & 4 & 1.5 \\
Adulto & 5 & 4 & 20 & 6 \\
Total & 30.5 & 24 & 29.5 \\
\hline
\end{tabular}

*Steffan presenta valores de Lycoriella mali (Fitch), pero es sinonimia de Lycoriella ingenua.

color amarillo claro semitransparente y brillante. A partir del segundo día se identifica la cabeza esclerotizada de la larva, el tercer día se observa la cabeza bien formada con sus mandíbulas. La cabeza de la larva puede ocupar hasta $20 \%$ de la superficie del huevo. Una hembra oviposita de 25 a 122 huevos (en promedio 81 huevos, $\mathrm{DE}=32.27$ ) individualmente o en grupos de 2 a 54. Frouz y Nováková (2001), indican que la hembra L. ingenua pone en promedio 53 huevos (rango 47-110), esta cantidad de huevos representa $35 \%$ menos que lo encontrado en el presente trabajo. O’Connor y Kiel (2005), encontraron que el número de huevos puestos por las hembras aumenta con el peso de estas. Los resultados de la media de la longitud y ancho del huevo, obtenidos en el presente estudio, son bajos comparados con los de Lewandowki et al. (2004), quienes encontraron que el huevo L. ingenua mide 0.29 $\mathrm{mm}$ de largo y $0.17 \mathrm{~mm}$ de ancho, similares resultados fueron hallados para B. impatiens midendo $0.24 \mathrm{~mm}$ de longitud y $0.16 \mathrm{~mm}$ de ancho (Mansilla, et al., 2001). Los resultados obtenidos en este estudio de las dimensiones promedio del huevo pueden deberse a que los diferentes estadios son en general de menor tamaño que los encontrados por Lewandowki et al. (2004).

\section{Larvas}

Las larvas de L. ingenua y B. impatiens presentan cabeza bien desarrolladas, cápsula de la cabeza negra; cuerpo filiforme con 12 segmentos, de color blanco transparente, se puede observar su sistema digestivo durante todos los estadios larvarios, presenta cápsula cefálica bien esclerotizada desde el primer estadio. Las larvas pasan por cuatro estadios larvarios, ultimo estadio larvario con aberturas ventiladoras en el primer segmento del tórax y siete aberturas ventiladoras abdominales, octavo segmento abdominal carente de aberturas ventiladoras. La descripción de las larvas de L. ingenua y B. impatiens concuerdan con las descripciones de larvas para la familia Sciaridae hechas por Mohrig y Menzel (2009) y Stefan (1981).

\section{Medias de estadios de L. ingenua}

En la tabla 2 se presentan las medias de longitud de los estadios de L. ingenua. Lewandowski et al. (2004), usando una dieta de turba y soja, obtuvieron los siguientes promedios de medidas de longitud para huevo 0.290 $\mathrm{mm}$; primer estadio larvario 0.782 , segundo estadio lar-

Tabla 2. Medias del largo de los diferentes estadios de $L$. ingenua $(\mathrm{mm})$.

\begin{tabular}{ccccc}
\hline Estadio & $\mathrm{N}$ & Rango & Promedio & $\mathrm{DE}$ \\
\hline Huevo & 66 & $0.126-0.243$ & 0.210 & 0.025 \\
Primer estadio & 54 & $0.419-0.966$ & 0.678 & 0.182 \\
Segundo estadio & 29 & $0.910-1.561$ & 1.283 & 0.191 \\
Tercer estadio & 33 & $1.734-3.681$ & 2.368 & 0.6043 \\
Cuarto estadio & 47 & $2.073-3.681$ & 3.632 & 0.885 \\
Pupa & 24 & $1.282-2.773$ & 1.803 & 0.4127 \\
\hline
\end{tabular}


vario 1.550 , tercer estadio larvario 2.802 , cuarto estadio larvario 5.519, pupa 2.539. En el presente estudio se obtuvieron medidas menores en todos los estadios, estas diferencias se pueden deber a la dieta usada para alimentar a las larvas y la variabilidad genética de la especie.

\section{Estadios larvarios de $B$. impatiens}

En la figura 3, se muestran los primeros 9 valores AWE $(k)$ $k=1,2 \ldots 9$; con base en los valores de AWE se selecciona como número de grupos $k^{*}$, aquel que maximiza $\operatorname{AWE}(k)$. Se infiere que las larvas de $B$. impatiens pasaron por cuatro estadios $\left(k^{*}=4\right)$ antes de prepupar. En las figuras $4 \mathrm{y}$ 5 , se grafican los cuatro grupos que se obtuvieron con el método de conglomerados jerárquicos. Los cuatro estadios larvarios de $B$. impatiens obtenidos con el procedimiento de conglomerados jerárquicos concuerdan con los encontrados para la familia Sciaridae por Steffan (1981). Mansilla et al. (2001) y Steffan (1974), también encontraron cuatro estadios para esta especie.

\section{Estadios larvarios de L. ingenua}

En la figura 6, se muestran las primeros 10 valores AWE que se obtuvieron: $k=1,2 \ldots 10$. De la figura 6 , se obtiene que el optimizador de los valores AWE se determina en $k^{*}$ = 4 estadios. Así, se concluyó que las larvas de L. ingenua pasan por cuatro estadios antes de prepupar. Los cuatro estadios larvarios obtenidos en este estudio, utilizando el método de conglomerados jerárquicos, concuerdan con los encontrados por otros autores que utilizaron caracteres morfológicos y mediciones de cápsula cefálica para separar los estadios larvales (Lewandowski et al., 2004; Frouz y Nováková, 2001; Steffan, 1981). En la figura 7 se muestran los cuatro grupos obtenidos por medio del método de conglomerados jerárquicos.

\section{Disposición de orificios ventiladores en larvas.}

El número y tipo de estadios por los que pasan las larvas concuerdan con los hallados por Steffan (1981) y Chapman (1982). Las larvas de primer estadio presentan un par de orificios ventiladores en el último segmento del abdomen por lo que son metapnéusticas, las larvas de segundo y tercer estadios son propnéusticas, presentan un solo par de orificios ventiladores en el primer segmento del tórax, y las larvas de cuarto estadio son hemipnéusticas, presentan un par de orificios ventiladores en cada uno de los primeros siete segmentos del abdomen y un par de orifi-

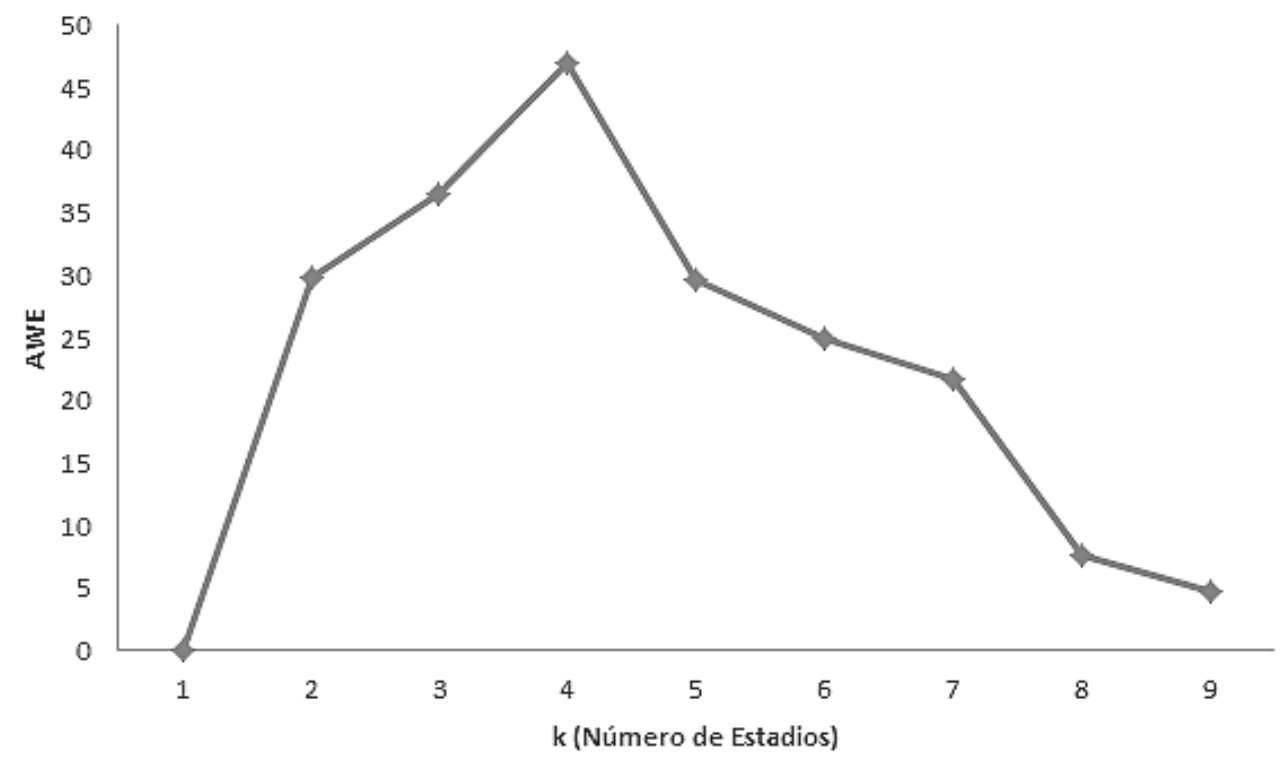

Figura 3. Valores AWE de la función mclust del S-PLUS obtenidos de las mediciones de amplitud de la capsula cefálica de la muestra de larvas de B. impatiens. 
cios ventiladores en el primer segmento del tórax. Sin embargo, este método no permite separar los estadios dos y tres. Para la separación de estadios larvarios se pueden usar métodos como la medición del largo y ancho de la cápsula cefálica (Lewandowski et al., 2004; Frouz y Nováková, 2001), aquí se consideró la longitud y ancho de la cápsula cefálica y longitud del cuerpo, combinado con el método de conglomerados jerárquicos, lo cual permite determinar de manera adecuada cada una de los estadios larvarios.

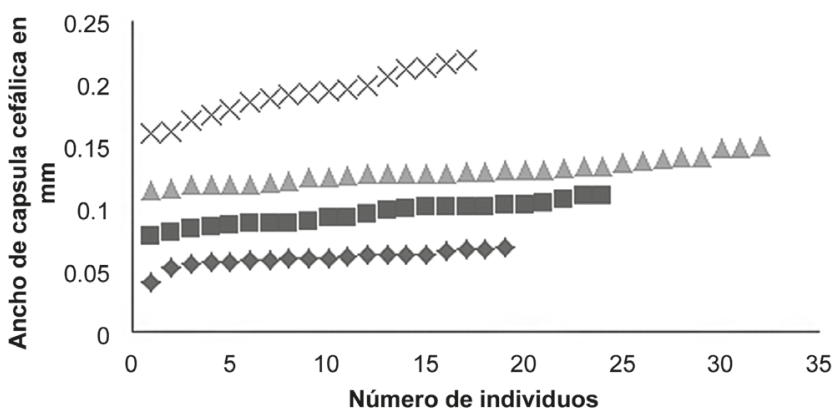

\section{Pupa}

La pupa de L. ingenua y B. Impatiens recién formada es de color blanco brillante; el segundo día cambia a color amarillo; después del tercer día es de color dorado brillante. La pupa es obtecta, apéndices unidos al cuerpo y cubiertos por una cutícula delgada. La pupa de L. Ingenua mide, en promedio, $1.8026 \mathrm{~mm}$ de largo y $0.5120 \mathrm{~mm}$ de ancho

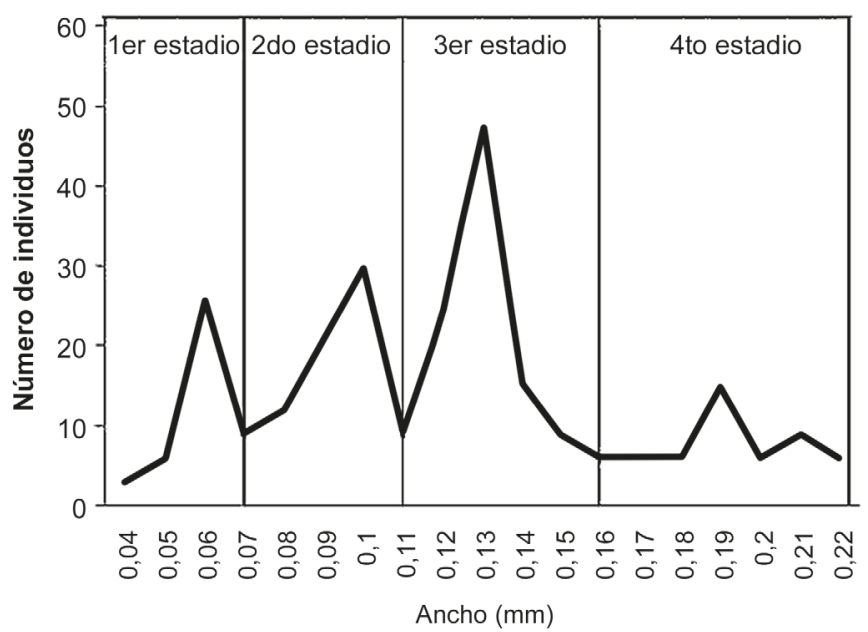

Figura 4. Estadios larvales de B. impatiens.

- Primer estadio, $\mathbf{m}$ segundo estadio, $\boldsymbol{\Delta}$ tercer estadio y $\mathbf{X}$ cuarto estadio.

Figura 5. Ancho de capsula cefálica por estadio de B. impatiens.

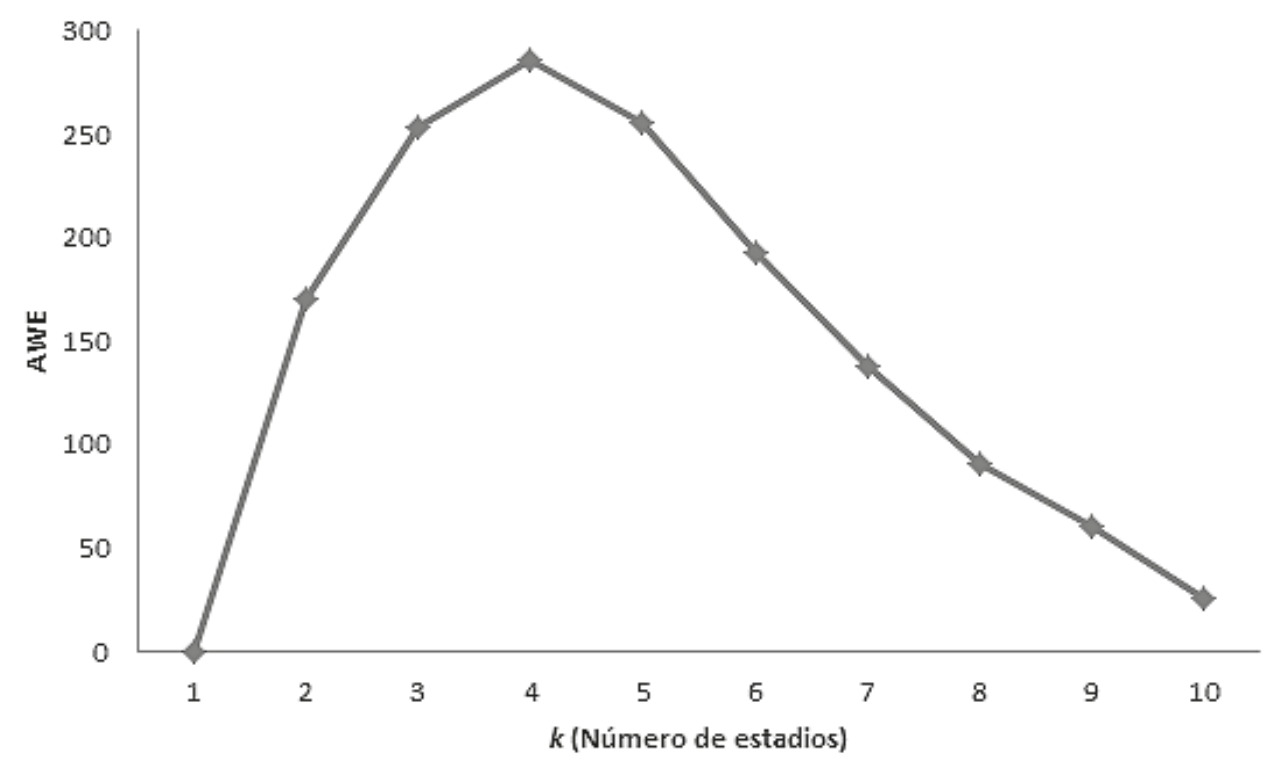

Figur a 6. Valores AWE obtenidos al agrupar con la amplitud de la cápsula cefálica y largo del cuerpo de la muestra de L. ingenua con el procedimiento de conglomerados jerárquicos. 


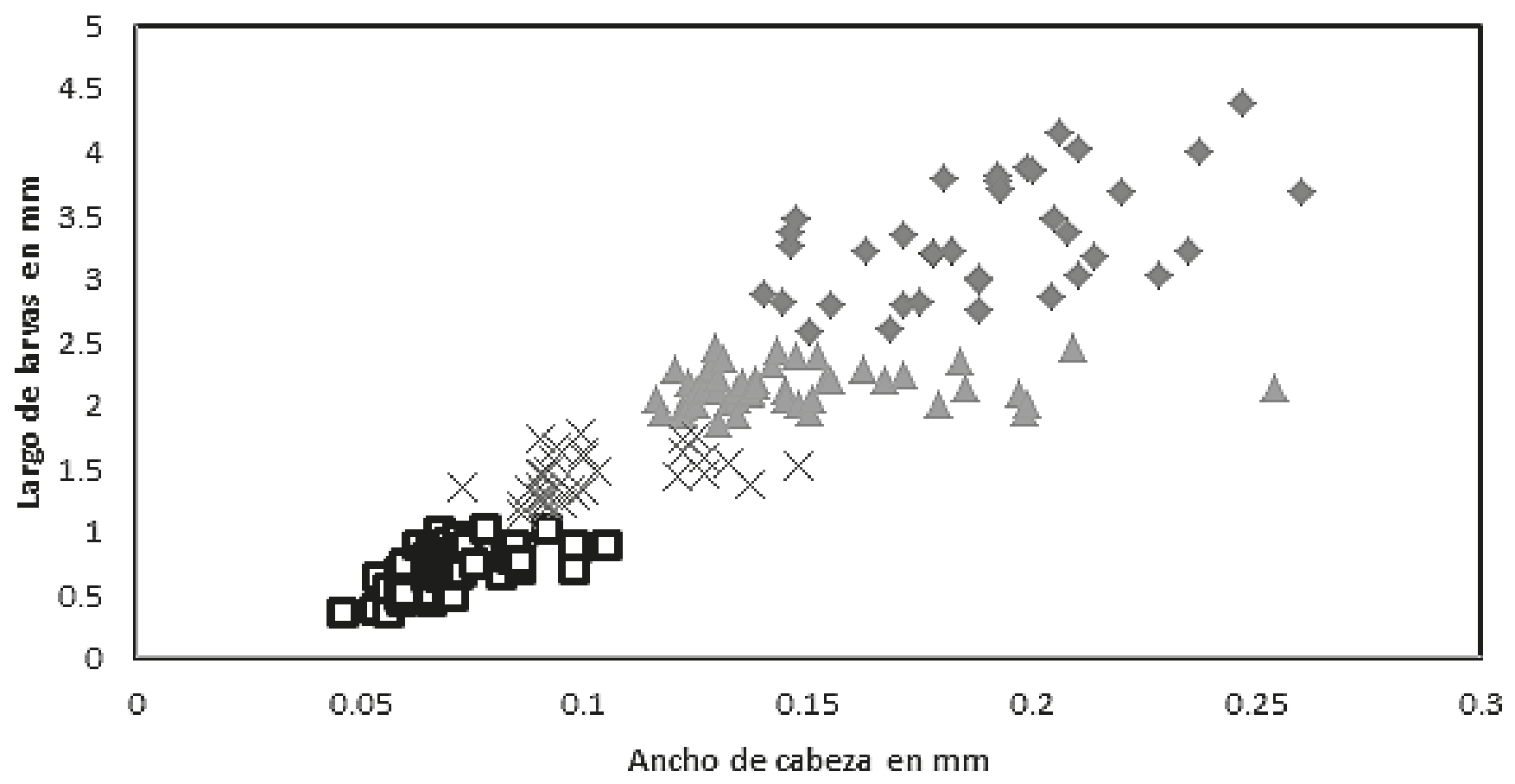

Figura 7. Representación gráfica de los 4 estadios larvales de L. ingenua obtenidos con conglomerados jerárquicos.

口 Primer estadio, $\mathrm{X}$ segundo estadio, $\boldsymbol{\Delta}$ tercer estadio $\mathrm{y} \bullet$ cuarto estadio.

(0.677-0.438 mm, DE $=0.061, \mathrm{n}=24)$. El promedio del largo de la pupa en este estudio es menor que el encontrado por Lewandowski et al. (2004), ellos indican un promedio del largo de la pupa de $2.539 \mathrm{~mm}(\mathrm{DE}=0.353)$. Sin embargo, en ambos estudios es similar la reducción en longitud de la larva de cuarto estadio a un poco más de la mitad de su tamaño cuando pasa al estado de pupa.

\section{Proporción de sexos}

Ambas especies presentaron en todos los ciclos machos y hembras, por lo que son disgénicas, la presencia de machos y hembras coinciden con lo encontrado por Steffan (1974) para L. ingenua y B. impateins. La proporción de sexos de L. ingenua después de analizar los genitales de 750 adultos fue de $44.40 \%$ machos (333 individuos) por $55.60 \%$ hembras (417 hembras). Por cada macho de L. ingenua se tuvieron 1.25 hembras. En el caso de B. impatiens se revisaron los genitales de 583 adultos, la proporción fue de $29.33 \%$ machos por $70.77 \%$ hembras (171 machos y 412 hembras). En B. impatiens por cada macho se tuvieron 2.41 hembras.

\section{Comportamiento}

Se encontró que en laboratorio y el vivero ambas especies se alimentan de las raíces de P. montezumae; sin embargo, los ciáridos en medios naturales se alimentan de hongos, materia orgánica en descomposición y raíces de plantas (Cibrián et al., 2008; Landis et al., 1989; Mohrig y Menzel, 2009; Pundt, 1999; Stefan, 1981). Las observaciones aquí realizadas, sugieren que $B$. impatiens y L. ingenua se han convertido en insectos plaga de importancia en los viveros forestales debido a los siguientes factores y condiciones: a) al no tener disponibilidad suficiente de hongos y materia orgánica en descomposición para alimentarse, pueden cambiar su dieta para alimentarse principalmente de raíces de $P$. montezumae, b) condiciones ambientales favorables en viveros (alta humedad, control de temperatura, sombra, refugio), c) casi nula presencia de enemigos naturales, d) gran capacidad reproductiva, e) uso generalizado de turba en el llenado de los tubetes en los viveros.

En la oviposición las hembras depositaron los huevos en las ranuras de las rodajas de papas. Las larvas de $L$. 
ingenua ocasionalmente pueden llegar a comer los cuerpos de los adultos muertos, larvas débiles o de instares menores, huevos y pupas que no han eclosionado vivos y muertos. De acuerdo con Steffan (1981), muchos ciáridos presentan este comportamiento. En condiciones de laboratorio este comportamiento se puede atribuir a que no encuentran otras fuentes de materia orgánica para alimentarse. Las larvas por lo general no construyen capullos, pero en ocasiones los hacen con excremento y basura. Además, las larvas llegan a sujetarse con hilos de seda antes de pupar. En este estudio se encontró que las larvas de cuarto instar de L. ingenua, cuando inician la prepupa, dejan de moverse y comer; contraen el cuerpo hasta reducirlo a un poco menos de $50 \%$ del tamaño original.

\section{Hongos asociados a B. impatiens y L. ingenua}

En este estudio se aislaron de las larvas de B. impatiens y L. ingenua los géneros de hongos, Verticillium Nees 1816, Penicillium Link 1809, Alternaria Nees 1816, Fusarium Link 1809, Paecilomyces Bainier 1907, Aspergillus P. Micheli 1729 y Mucor P. Micheli ex L. 1753. De los adultos de ambas especies de mosquito fungoso negro se aislaron cinco géneros, Verticillium, Penicillium, Alternaria, Fusarium y Aspergillus. En condiciones de laboratorio las larvas se alimentaron de los siete géneros de hongos aislados, este hábito concuerda con el observado en condiciones de campo, donde las larvas de ciáridos se alimentan de hongos y materia orgánica en descomposición (Mohrig y Menzel, 2009; Pundt, 1999; Stefan, 1981). En este estudio se encontró la especie Fusarium circinatum Nirenberg et O’Donnell, el cual es un patógeno importante del género Pinus. (Wingfield, et al., 2008). Las secuencias genéticas de este hongo se depositaron en GenBank con el número de acceso KM275219. Los adultos y larvas de ciáridos pueden ser medio de transporte de hongos patógenos y no patógenos (Gardiner, 1990; Hurley et al., 2007, 2010; James et al., 1995; Kalb y Millar, 1986; Pundt, 1999; Shamshad et al., 2009). Los adultos son muy activos en el vivero y de esta manera dispersan los hongos; son acarreadores de Botrytis cinerea, Fusarium y Phoma (James et al., 1995; Keates et al., 1989; Mansilla et al., 2001).
En el vivero de Temamatla los adultos de ciáridos son los responsables de diseminar F. circinatum en las platabandas y las larvas pueden ser transmisores que inoculan las plántulas de pino, ya que en su integumento llevan a este patógeno. La forma de transmitir el hongo a la raíz es por medio de la alimentación de las larvas las cuales hacen heridas a las raíces del pino, por donde penetran los hongos. Además, las larvas por medio de su cuerpo introducen al hongo en la raíz (James et al., 1995; Pundt, 1999; Springer, 1995b). Sin embargo, en Sudáfrica en viveros forestales de coníferas, se encontró que $B$. impatiens puede que no tenga un rol importante en la movilidad de los hongos patógenos (Hurley et al., 2007).

\section{Daño en raíz de plántulas de Pinus montezumae}

Las larvas atacaron la raíz principal del P. montezumae. Primero empiezan por comer la rizodermis, después perforan la exodermis, que es el tejido de protección de la raíz (James, 2003). La herida causada en la raíz es la puerta de entrada para diversos patógenos. Las larvas se instalan en el córtex y consumen el parénquima cortical, que es una zona rica en reservas de la raíz (James, 2003; Mirov, 1967). Las larvas se mueven dentro del córtex de manera vertical sin perforar la exodemis que sirve como protección. La exodermis conserva la humedad dentro de la raíz y crea un microclima con condiciones adecuadas para el desarrollo de las larvas y el crecimiento de hongos. Las larvas detienen su consumo de la raíz en la endodermis, que es un tejido de protección y que puede estar lignificado; sin embargo, cuando este comienza a pudrirse es consumido por las larvas. Por último, se alimentan del xilema y el floema. De esta manera queda la raíz como un tubo, con un centro que está formado por la medula lignificada y una parte exterior que constituye la exodermis lignificada. Así, dentro de este tubo las larvas se mueven libremente, principalmente hacia el cuello de la raíz. Springer (1995a) observó un comportamiento similar en larvas del mosquito fungoso negro (Bradysia sp.) que se alimentan de raíces de Trifolium incarnatum L., estas consumen la epidermis y el córtex, los tejidos vasculares lignificados no son consumidos. En este estudio se observó 
que las plántulas de P. montezumae, al no tener una apropiada comunicación con la raíz, presentan síntomas de daños en la parte aérea que se manifiestan como marchitez, escaso crecimiento, pérdida de acículas y de vigor. Los síntomas pueden ser confundidos con alguna enfermedad causada por hongos (Cibrián et al., 2008).

Las raíces concentran el ataque de larvas en los primeros $3 \mathrm{~cm}$ a $5 \mathrm{~cm}$ partiendo del cuello de la raíz hacia la cofia. Esta zona corresponde en las plántulas al área de maduración de la raíz, lugar de almacenamiento de reservas. El mayor número de larvas dentro de la raíz se encontró en esta zona. Hasta 35 larvas por raíz de todos los instares.

\section{Atracción de raíces a larvas}

En el laboratorio se demostró que las larvas perciben la ubicación de raíces, probablemente por estímulos olfativos; el tiempo que las larvas necesitaron para reconocer y trasladarse a la raíz en las cajas Petri fue de 15 minutos en promedio $(\mathrm{DE}=10)$; la mayoría se alimentó del tejido vegetal tierno e hicieron un daño similar al descrito arriba.

\section{CONCLUSIONES}

Por primera vez en México se identifica a $B$. impatiens y $L$. ingenua en viveros forestales atacando plántulas de coníferas. El ciclo biológico de B. impatiens a $21^{\circ} \mathrm{C} \pm 2{ }^{\circ} \mathrm{C}$ en laboratorio fue de 27.5 días con cuatro instares larvarios. El ancho de la capsula cefálica es un parámetro que sirve para separar los instares larvarios. La proporción sexual fue de 2.4 hembras por cada macho. El ciclo biológico de $L$. ingenua a $21{ }^{\circ} \mathrm{C} \pm 2{ }^{\circ} \mathrm{C}$ en laboratorio fue 30.5 días, presenta cuatro instares larvarios, los mejores parámetros para separarlos son el ancho de la cápsula cefálica y largo del cuerpo en combinación. La proporción sexual fue de 1.25 hembras por cada macho. La duración del ciclo biológico de estas especies de Sciaridae, está estrechamente relacionada con la temperatura y la dieta.

En el vivero forestal de Temamatla, Estado de México las larvas y adultos de L. ingenua y B. impatiens se asociaron con los géneros de hongos fitopatógenos Vertici- llium, Penicillium, Alternaria, Fusarium, Paecilomyces, Aspergillus y Mucor. Estos hongos pueden ser distribuidos dentro del sustrato de los tubetes por las larvas y entre contenedores y platabandas por los adultos. Las larvas de B. impatiens y L. ingenua se asocian a Fusarium circinatum que es un patógeno importante del género Pinus.

Los daños causados en la raíz por las larvas del ciáridos pueden ocasionar la muerte de las plántulas de $P$. montezumae. Las larvas de mosquito fungoso negro son capaces de detectar en 15 minutos la raíz del pino. La asociación de $B$. impatiens y L. ingenua con hongos patógenos y su posible transmisión a las plántulas del género Pinus debe de ser estudiada para conocer con precisión el papel que tienen estos insectos en la movilidad y transmisión de hongos patógenos de importancia forestal.

\section{AGRADECIMIENTOS}

Al Mayor de Caballería Teodoro Ariel Ake Dzib, Jefe del Vivero Forestal Militar Temamatla; a los Ingenieros Alberto González Enríquez, Oscar Andree Azuara González y Alejandro Castro Moreno, personal técnico del Vivero de Temamatla, por su apoyo en la fase de campo en el control del mosquito fungoso negro.

\section{REFERENCIAS}

Aguilera, P.A. y K.F. Ortega. 1996. Bradysia coprophila (Lintner) (Diptera: Sciaridae) en Trébol Rosado (Trifolium pratense L.). Agricultura Técnica 56(2):135-138.

Braun, S.E., J.P. Sanderson, E.B. Nelson, M.L. Daughtrey y S.P. Wraight. 2009. Fungus gnat feeding and mechanical wounding inhibit Pythium aphanidermatum infection of geranium seedlings. Phytopathology 99(12):1421-1428.

Carrillo, L. 2003. Los hongos de los alimentos y forrajes. Universidad de Salta. Salta, Argentina. 130 pp.

Chapman, R.F. 1982. The insects structure and function. Harvard University Press. Cambridge, Massachusetts. EUA. 919 p.

Cibrián T., D., D.S. García y M.B. Don Juan. 2008. Manual de identificación y manejo de plagas y enfermedades en germoplasma y planta producida en viveros. Comisión Nacional Forestal. México. 153 p. 
Erler, F., E. Polat, H. Demir, M. Catal y G. Tuna. 2011. Control of mushroom sciarid fly Lycoriella ingenua populations with insect growth regulators applied by soil drench. Journal of Economic Entomology 104(3):839-844.

Frouz, A. y A. Nováková. 2001. A new method for rearing the sciarid fly, Lycoriella ingenua (Diptera: Sciaridae), in the laboratory: possible implications for the study of fly-fungal interactions. Pedobiologia 45(4):329-340.

García P., F. 2008. Fungus gnat: Insecto plaga en ornamentales. Instituto Nacional de investigaciones Forestales, Agrícolas y Pecuarias (Inifap). $6 \mathrm{p}$.

Gardiner, R.B., W.R. Jarvis y J.L. Shipp. 1990. Ingestion of Phytium spp. by larvae of the fungus gnat Bradysia impatiens (Diptera:Sciaridae). Annals of Applied Biology 116(2): 205-212.

Gillespie, R.D. y J.G. Menzies. 1993. Fungus gnats vector Fusarium oxysporum f.sp. radicis-lycopersici. Annals of Applied Biology 123(3): 539-544.

Hurley, B.P., B. Slippers, T.A. Coutinho, B.D. Wingfield, P. Govender y M.J. Wingfield. 2007. Molecular detection of fungi carried by Bradysia difformis (Sciaridae: Diptera) in South African forestry nurseries. Southern Hemisphere Forestry Journal 69(2):103-109.

Hurley, B.P., B. Slippers, B.D. Wingfield, P. Govender, J.E. Smith y M.J. Wingfield. 2010. Genetic diversity of Bradysia difformis (Sciaridae: Diptera) populations reflects movement of an invasive insect between forestry nurseries. Biological Invasions 12(4):729-733.

James, D.M. 2003. Botany an introduction to plant biology. 3a ed. Jones and Bartlett Publishers. EUA. Massachusetts. 848 p.

James, R.L., R.K. Dumroese y D.L. Wenny. 1995. Botritys cinerea carried by adult fungus gnats (Diptera: Sciaridae) in container nurseries. Tree Planters Notes 46(2):48-53.

Kalb, D.W. y R.L. Millar. 1986. Dispersal of Verticillium alboatrum by the fungus gnat (Bradysia impatiens). Plant Disease 70(8):752-753.

Keates, S.E., R.N. Sturrock y J.R. Sutherland. 1989. Populations of adult fungus gnats and shores flies in British Columbia container nurseries as relates to nursery environment, and incidence of fungi on the insects. New Forests 3:1-9.
Komarov, S.S. 2009. A review of species of the sciarid-fly genus Lycoriella Frey (Diptera, Sciaridae) of the Altai fauna. Entomological Review 89(2):175-180.

Landis, T.D., R.W. Tinus, S.E. McDonald y J.P. Barnett. 1989. The biological component: Nursery Pests and Mycorrhizae. Vol. 5. The Container Tree Nursery Manual. Agric. Handbk. 674. Washington, DC: U.S. Department of Agriculture, Forest Service. $171 \mathrm{p}$

Lewandowski, M., A. Sznyk y A. Bednarek. 2004. Biology and morphometry of Lycoriella ingenua (Dipetera: Sciaridae). Biology Letters 41(1):41-50.

Loudon, B.J. 1978. A new species of Lycoriella Frey (Diptera: Sciaridae) infesting cultivates mushrooms in New South Wales. Journal of the Australian Entomological Society 17(2): 163-166.

Mansilla, J. P., M. I. Pastoriza y R. Pérez. 2001. Estudio sobre la biología y control de Bradysia paupera Tuomikoski (=Bradysia difformis Frey) (Diptera: Sciaridae). Boletín de Sanidad Vegetal. Plagas 27:411-417.

Menzel, F., J.E. Smith y J.P. Chandler. 2006. The sciarid fauna of the British Isles (Diptera: Sciriadae), including descriptions of six new species. Zoological Journal of the Linnean Society 146(1):1-147.

Menzel, F., J. E. Smith y B. N. Colauto. 2003. Bradysia difformis Frey and Bradysia ocellaris (Comstock): two additional neotropical species of black fungus gnats (Dipetera: Sciaridae) of economic importance: a redescription and review. Annals of the Entomological Society of America 96(4):448-457.

Mirov, N.T. 1967. The genus Pinus. The Ronald Press Company. EUA, Nueva York. 602 p.

Mohrig, W. y F. Menzel. 2009. Sciaridae (Black fungus gnats). In: B.V. Brown, A. Borkent, J. M. Cumming, D. M. Wood, N. E. Woodley y M. A. Zumbado, eds. Manual of Central American Diptera Vol 1. Monograph Publishing Program. Canada: National Research Council of Canada. p:279-292.

Mohrig, W., K. Helelr, H. Hippa, P. Vilkamaa y F. Menzel. 2012. Revision of black fungus gnats (Diptera: Sciaridae) of North America. Studia Dipterologica 19(1/2):141286. 
O’Connor, L. y C.B. Keil. 2005. Mushroom host influence on Lycoriella mali (Diptera: Sciaridae) life cicle. Journal of Economic Entomology 98(2):342-349.

Pérez M., C., R.I. Rojas M., R. Alatorre R., F. Hernández R. y G. Otero C. 2013. Análisis de la variación intraespecífica de Conidiobolus coronatus usando RAPD y secuencias ITS. Enfermedades Infecciosas y Microbiología Clínica $30(20): 1-6$.

Poinar, G.O. JR. y G.M. Thomas. 1984. Laboratory guide to insect pathogens and parasites. Plenum Press. Nueva York y Londres. 392 p.

Pundt, L. 1999. Fungus gnats are a serious pests. Yankee Grower Septiembre-Octubre.

Radin, B., W.V.R. Dos-Santos, L.B. Brito, S. Witter y S.J.R. Pfeifer. 2009. Bradysia sp. em morangueiro. Ciencia Rural 39(2):547-550.

Shamshad, A., A.D. Clift y S. Mansfield. 2009. The effect of tibia morphology on vector competency of mushroom sciarid flies. Journal of Applied Entomology 133(6):484490.

Shin, S.G., H.S. Lee y S. Lee. 2012. Dark winged fungus gnats (Diptera: Sciaridae) collected from shiitake mushroom in Korea. Journal of Asia-Pacific Entomology 15(1):174181.

Smith, J.E., P.F. White, R.N. Edmondson y D. Chandler. 2006. Effect of different Agaricus species on the development of the mushroom sciarid fly Lycoriella ingenua. Entomologia Experimentalis et applicata 120(1):63-69.

S-PLUS 6.1 for Windows Guide to Statistics. 2001. Vol. 2. Insightful Corporation, Seattle, WA. p:141-146.
Springer, T.L. 1995a. Fungus gnats (Diptera: Sciaridae) feeding damage to legume seedling. Journal of the Kansas Entomological Society 68(2):240-242.

Springer, T.L. 1995b. Vulnerability of pasture and range legumes to fungus gnats. Crop Science 35(2):534-536.

Steffan, W.A. 1981. Sciaridae. In: J.F. McAlpine, B.V. Peterson, G.E. Shewell, H.J. Teskey, J.R. Vockeroth y D.M. Wood, eds. Manual of Neartic Diptera, vol. 1. Research Branch Agriculture Canada Monograph 27. Canada. p:247-255.

Steffan, W.A. 1974. Laboratory studies and ecological notes on Hawaiian Sciaridae (Diptera). Pacific Insects 16(1):41-50.

Wilkinson, J.D. y D.M. Daugherty. 1970. The biology and immature stages of Bradysia impatiens (Diptera: Sciaridae). Annals of the Entomological Society of America 63(3):656-660.

Wingfield, M.J., A. Hammerbancher, R.J. Ganley, E.T. Steenkamp, T.R. Gordon, B.D. Wingfield y T.A. Coutinho. 2008. Pitch canker by Fusarium circinatum a growing threat to pine plantations and forest worldwide. Australasian Plant Pathology 37:319-334.

White, P.F., J.E. Smith y F. Menzel. 2000. Distribution of Sciaridae (Dipt.) species infesting commercial mushroom farms in Britain. Entomologist's Monthly Magazine 136(1636/1639:207-209.

Manuscrito recibido el 8 de noviembre de 2013.

Aceptado el 7 de agosto de 2014.

Este documento se debe citar como:

Marín-Cruz, V.H., D. Cibrián-Tovar, J.T. Méndez-Montiel, O.A. PérezVera, J.A. Cadena-Meneses, H. Huerta, G. Rodríguez-Yam y J.A. Cruz-Rodríguez. 2015. Biología de Lycoriella ingenua y Bradysia impatiens (Diptera: Sciaridae). Madera y Bosques 21(1):113-128. 